\title{
The complex of PAMAM-OH dendrimer with Angiotensin (I-7) prevented the disuse-induced skeletal muscle atrophy in mice
}

This article was published in the following Dove Press journal:

International Journal of Nanomedicine

13 March 2017

Number of times this article has been viewed

\author{
Valeria Márquez-Miranda ${ }^{1,2, *}$ \\ Johanna Abrigo $3,4, *$ \\ Juan Carlos Rivera ${ }^{3,4}$ \\ Ingrid Araya-Durán' \\ Javier Aravena ${ }^{3,4}$ \\ Felipe Simon ${ }^{3,4}$ \\ Nicolás Pacheco' \\ Fernando Danilo \\ González-Nilo 1,2,5 \\ Claudio Cabello-Verrugio 3,4 \\ 'Center for Bioinformatics and \\ Integrative Biology (CBIB), Facultad \\ de Ciencias Biologicas, Universidad \\ Andres Bello, Santiago, ${ }^{2}$ Fundación \\ Fraunhofer Chile Research, Las \\ Condes, ${ }^{3}$ Departamento de Ciencias \\ Biologicas, Facultad de Ciencias \\ Biologicas \& Facultad de Medicina, \\ Universidad Andres Bello, ${ }^{4}$ Millennium \\ Institute on Immunology and \\ Immunotherapy, Santiago, ${ }^{5}$ Centro \\ Interdisciplinario de Neurociencia \\ de Valparaíso, Facultad de Ciencias, \\ Universidad de Valparaíso, Valparaíso, \\ Chile
}

*These authors contributed equally to this work

Correspondence: Fernando Danilo González-Nilo

Facultad de Ciencias Biologicas, Center for Bioinformatics and Integrative Biology (CBIB), Universidad Andres Bello, Avenida Republica 239, Santiago 8370146, Chile

Email fernando.gonzalez@unab.cl

Claudio Cabello-Verrugio Departamento de Ciencias Biologicas, Facultad de Ciencias Biologicas, Universidad Andres Bello, Avenida República 239, Santiago 8370I46, Chile Email claudio.cabello@unab.cl

\begin{abstract}
Angiotensin (1-7) (Ang-(1-7)) is a bioactive heptapeptide with a short half-life and has beneficial effects in several tissues - among them, skeletal muscle - by preventing muscle atrophy. Dendrimers are promising vehicles for the protection and transport of numerous bioactive molecules. This work explored the use of a neutral, non-cytotoxic hydroxyl-terminated poly(amidoamine) (PAMAM-OH) dendrimer as an Ang-(1-7) carrier. Bioinformatics analysis showed that the Ang-(1-7)-binding capacity of the dendrimer presented a 2:1 molar ratio. Molecular dynamics simulation analysis revealed the capacity of neutral PAMAM-OH to protect Ang-(1-7) and form stable complexes. The peptide coverage ability of the dendrimer was between $\sim 50 \%$ and $65 \%$. Furthermore, an electrophoretic mobility shift assay demonstrated that neutral PAMAM-OH effectively bonded peptides. Experimental results showed that the Ang-(1-7)/PAMAM-OH complex, but not Ang-(1-7) alone, had an anti-atrophic effect when administered intraperitoneally, as evaluated by muscle strength, fiber diameter, myofibrillar protein levels, and atrogin-1 and MuRF-1 expressions. The results of the Ang-(1-7)/PAMAM-OH complex being intraperitoneally injected were similar to the results obtained when Ang-(1-7) was systemically administered through mini-osmotic pumps. Together, the results suggest that Ang-(1-7) can be protected for PAMAM-OH when this complex is intraperitoneally injected. Therefore, the Ang-(1-7)/PAMAM-OH complex is an efficient delivery method for Ang-(1-7), since it improves the anti-atrophic activity of this peptide in skeletal muscle.
\end{abstract}

Keywords: muscle wasting, peptide delivery, carrier, anti-atrophic peptide

\section{Introduction}

The biological activities of several proteins and peptides can be exploited for therapeutic applications. Furthermore, the controlled release of oligopeptides has many potential applications in biomedical and pharmaceutical areas, including in the treatment of diabetes, cancer and tumors, and metabolic, cardiovascular, and infectious diseases. However, peptide use is limited due to low hydrolytic stability, poor systemic distribution, short half-life resulting from rapid metabolization, and low ability to cross physiological barriers.

Consequently, many peptide-delivery strategies have been studied, including direct injection into the malignant tissue and administration using osmotic pumps. Alternatively, hyperbranched polymers and dendrimers exhibit properties that make them attractive candidates for oligopeptide delivery. The chemical properties of dendrimer terminal groups and branch flexibilities play critical roles in interactions with cargo molecules. Indeed, different poly(amidoamine) (PAMAM) dendrimer terminal groups are involved, 
to varying degrees, in interactions with different drug types, as previously demonstrated through molecular modeling tools and experimental validation. ${ }^{1,2}$ Therefore, choosing the right terminal moiety is critical for modulating the delivery (ie, kinetics parameters) and protection of drug-like peptides.

Computational chemistry and cheminformatics are required for rational molecular designing and discovering new chemical groups from small-molecule databases. Related to this, a new bioinformatics-based strategy for the rational design of new dendrimers as DNA carriers that employs the knowledge of protein-DNA interactions was recently reported. ${ }^{3}$ Among the dendrimers with potential applications, hydroxyl-terminated PAMAM (PAMAM-OH) dendrimers are suggested safe drug carriers due to non-toxic effects observed during in vitro and in vivo applications. ${ }^{4}$ Furthermore, the PAMAM-OH dendrimer can preferentially distribute in the brain, enabling targeted therapeutic delivery in disorders such as cerebral palsy, Alzheimer's disease, and multiple sclerosis. ${ }^{4,5}$ In summary, our concept for dendrimerbased nano-carriers combines various methodological advantages, including efficient cell interactions due to nanosize scaling, a benefit not afforded by liposomes; a monodisperse structure that confers higher stability than micelle-based carriers; a high loading capacity of cargo molecules; and a high ability to cross cellular barriers.

Among the various therapeutic applications for drug-like peptides, angiotensin 1-7 (Ang-(1-7)) is highly beneficial in the treatment of circulatory system, skeletal muscle, and nervous system diseases. ${ }^{6}$ In particular, skeletal muscle atrophy is a loss of muscle mass and strength, a process that is characterized by decreased muscle fiber diameter, fewer myofibrillar proteins, such as myosin heavy chain (MHC), increased proteolytic pathway dependent on the ubiquitin proteasome system, and changes in muscle fiber types. ${ }^{7}$ One factor that produces muscle atrophy is angiotensin II (Ang II). ${ }^{8-10}$ In several tissues, including skeletal muscle, Ang II and Ang-(1-7) present opposite functions. ${ }^{11-14}$ Related to this, a recent report described the effect of Ang-(1-7) as an anti-atrophic factor in skeletal muscle in Ang II-dependent and -independent models, such as in immobilization or sepsis. ${ }^{15-18}$ Therefore, finding the mechanisms that potentiate the anti-atrophic actions of Ang-(1-7) is relevant in a therapeutic context. However, the instability of Ang-(1-7) restricts efficient oral or injected administration. ${ }^{11,19}$ A new delivery method is needed that increases the half-life and improves the bioavailability of this peptide in target tissues.

This study explored the use of a PAMAM-OH dendrimer as an Ang-(1-7) carrier for the treatment of skeletal muscle atrophy. To determine the stability of the peptide/dendrimer formulation, molecular dynamics (MD) simulations were conducted to elucidate the structural phenomena involved in the interaction between both macromolecules and their controlled release into cells. Once a stable peptide/dendrimer formulation was established, in vivo studies were performed to evaluate the impact of the dendrimer on the short-life increase of peptides. To accomplish this, a model of disuse by immobilization (ie, unilateral hind limb casting) was used to evaluate Ang-(1-7)/PAMAM-OH complex stability in mice treated with intraperitoneal (IP) injections of Ang-(1-7) or the Ang-(1-7)/PAMAM-OH complex.

\section{Methods \\ Molecular simulations}

The fourth-generation neutral PAMAM-OH dendrimer molecular model (Figure S1A) was built by dividing the dendrimer into three parts: the ethylenediamine core, internal dendrons, and the surface, which consisted of hydroxyl terminal groups. Each section was parameterized separately, taking into consideration the CHARMM General force field ${ }^{20}$ and PARAMCHEM platform. ${ }^{21,22}$ Components were then connected to form a whole dendrimer by employing homemade scripts. Meanwhile, the Ang-(1-7), molecular model, sequence DRVYIHP (Figure S1B), was obtained using the Molefacture Protein Builder plugin of Visual Molecular Dynamics software. ${ }^{23}$ The peptide topology, parameters, and charges were obtained from the CHARMM27 protein force field. ${ }^{24}$

Each structure was placed separately into a TIP $3 \mathrm{P}^{25}$ water box and then subjected to energy minimization using NAMD software ${ }^{26}$ and the conjugate gradient algorithm until reaching convergence. Subsequently, both systems were equilibrated by MD simulations to obtain relaxed structures.

Afterward, molecular docking simulations were performed using the AutoDock4.2 suite $^{27}$ to obtain a peptide/ dendrimer complex. The partial charges were assigned using the CHARMM force field. In this way, three Ang-(1-7) peptides were docked separately in the interior of dendrimer cavities. The center of the grid box was chosen so that the ligand search space was close to the dendrimer ethylenediamine core. Considering the size of the peptide, only three peptides were coupled to the dendrimer. Grid points were set in the $\mathrm{x}, \mathrm{y}$, and $\mathrm{z}$ dimensions, considering a box size of $25 \AA \times 25 \AA \times 25 \AA$. Grid spacing was set to $0.375 \AA$. Nonpolar hydrogens were not considered in these simulations. The optimal conformation was chosen according to the best score in each docking. 
Peptide/dendrimer complexes were then placed into a TIP3P water box with a salt concentration of $150 \mathrm{mM} \mathrm{NaCl}$ to simulate physiological conditions. The details of the systems are shown in Table S1.

After appropriate energy minimization stage of peptide/dendrimer system, to study the molecular behavior and interaction between neutral PAMAM-OH and Ang-(1-7) peptide, MD simulation was carried out by $60 \mathrm{~ns}$.

All MD simulations described in this study were run under the isobaric-isothermal number of particles, constant pressure, constant temperature (NPT) ensemble $(\mathrm{T}=310 \mathrm{~K}$; $\mathrm{P}=1 \mathrm{~atm}$ ), with periodic boundary conditions in all three directions using the NAMD 2.9 package. ${ }^{26}$ To maintain constant pressure and temperature, Langevin dynamics with a damping coefficient of $1 \mathrm{ps}$ and the Nose-Hoover Langevin piston method were applied. ${ }^{28}$ All hydrogen bonds were constrained during the MD simulations using the SHAKE algorithm. Long-range electrostatic interactions were calculated with the particle mesh Ewald ${ }^{29}$ algorithm, and van der Waals (vdW) forces were estimated using a cutoff of $10 \AA$. Equations of motion were integrated with a time step of $2 \mathrm{fs}$ using the Verlet algorithm.

\section{Electrophoresis measurements}

Complexes were formed in water from the Mili- $\mathrm{Q}^{\circledR}$ Integral Water Purification System (EMD Millipore Corp., Billerica, MA, USA) at a $\mathrm{pH}$ of $7.4(\mathrm{NaOH})$ by adding PAMAM-OH (Dendritech Inc., Midland, MI, USA) to the Ang-(1-7) peptide (Sigma-Aldrich, St Louis, MO, USA) solutions at different peptide/dendrimer molar ratios that were then vortexed and incubated for $30 \mathrm{~min}$ at room temperature $\left(\sim 25^{\circ} \mathrm{C}\right)$. Complex formation between PAMAM-OH and Ang-(1-7) was followed by an electrophoretic shift assay. Peptide/dendrimer complexes $(10 \mu \mathrm{L})$ were mixed with $3 \mu \mathrm{L}$ Coomassie blue g-250 (Sigma-Aldrich) staining solution for $15 \mathrm{~min}$ at room temperature. Complex formation was assessed by migration retardation in the electrophoresis on a $5 \%$ agarose gel for $1 \mathrm{~h}$ at $100 \mathrm{~V}$ using the Bio-Rad Mini-Sub ${ }^{\circledR}$ Cell GT DNA electrophoresis system (Bio-Rad Laboratories Inc., Hercules, CA, USA). After electrophoresis was performed, the resulting gel was analyzed under white light.

\section{Animals}

Twelve-week-old C57BL/10J male mice (triplicate independent experiments were performed with six mice per group) were used for experimentation. Unilateral immobilization was performed in the lower hind limb for different times using a $3 \mathrm{M}^{\mathrm{TM}}$ Scotchcast ${ }^{\mathrm{TM}}$ Soft Cast Casting Tape. ${ }^{17}$ The treatments were performed for $24 \mathrm{~h}$ and $14 \mathrm{~d}$ depending on the evaluated parameter. For IP treatment, $50 \mu \mathrm{L}$ of phosphate-buffered saline (PBS), Ang-(1-7) (0.8 mM), PAMAM-OH (0.4 mM), and the complex Ang-(1-7)/PAMAM-OH (2:1) were IP injected three times a week starting $24 \mathrm{~h}$ before cast immobilization. For osmotic pump delivery, PBS, Ang-(1-7) (100 ng/kg/min), PAMAM-OH (1 ng/kg/min), and the Ang(1-7)/PAMAM-OH complex (2:1 molar ratio) were osmotically infused through micropumps (Alzet-Durect, Cupertino, CA, USA), as previously described, ${ }^{15-18} 24 \mathrm{~h}$ before cast immobilization. After experimentation, the animals were euthanized under anesthesia and the gastrocnemius (GAST) muscles were dissected, removed, and rapidly frozen and stored at $-80^{\circ} \mathrm{C}$ until processing. All protocols were conducted in strict accordance and with the formal approval of the Animal Ethics Committee at the Universidad Andres Bello.

\section{Muscle histology and muscle fiber determination and quantification}

Freshly frozen GAST muscles were cryosectioned $(7 \mu \mathrm{m})$ and stained with hematoxylin and eosin (H\&E) or Alexa-Fluor 594-tagged wheat germ agglutinin (WGA) (Life Technologies, Carlsbad, CA, USA) according to standard procedures. Fiber sizes were determined by WGA staining using the ImageJ software (National Institutes of Health, Bethesda, MD, USA) on seven blindly selected random images of each experimental condition. Fibers were manually selected, and the minimal Feret diameter of each fiber was estimated using the ImageJ software. ${ }^{17,30}$

\section{Contractile properties}

After treatment, the mice were anesthetized and the insertional tendon (calcaneal) of the GAST was isolated, tied with surgical silk suture, and cut from its insertion. Then, the leg was removed by transecting the femur to maintain intact the origin of the GAST in order to minimize muscle damage due to dissection. The leg, with attached GAST, was tied firmly with surgical silk and immediately placed in a dish containing oxygenated Krebs-Ringer solution. Then the muscles were transferred to a custom-built Plexiglas bath filled with oxygenated Krebs-Ringer solution thermostatically maintained at $37^{\circ} \mathrm{C}$ for optimal oxygen diffusion. The muscles were tied to a MLT 1030/D force transducer (AD Instruments, Dunedin, New Zealand). Muscle strength was determined as described previously. ${ }^{31-33}$ Briefly, the optimum muscle length (Lo) and stimulation voltage were determined from micromanipulations of muscle length to produce the maximum isometric twitch force using a S48 
Stimulator (Grass Research Instruments, West Warwick, RI, USA), controlled and measured using a Power Lab 4/35 (AD Instruments) and computed with Lab Chart analysis software (AD Instruments). Maximum isometric specific tetanic force was determined from the plateau of the curve of relationship between specific isometric force $\left(\mathrm{mN} / \mathrm{mm}^{2}\right)$ with a stimulation frequency in the range $1-200 \mathrm{~Hz}$ for $450 \mathrm{~ms}$, with 2 min of rest between stimuli, and muscle mass and Lo were used to calculate it.

\section{RNA isolation, reverse transcription, and quantitative real-time polymerase chain reaction (PCR)}

Total RNA isolation and cDNA reverse transcription were performed as previously described. ${ }^{18}$ Quantitative real-time PCR reactions were performed in triplicate using an Eco Real-Time PCR System (Illumina, San Diego, CA, USA) with predesigned primer sets for mouse atrogin-1, MuRF-1, and the housekeeping gene tubulin (TaqMan Assays-onDemand; Applied Biosystems, Foster City, CA, USA). The messenger RNA (mRNA) expression was quantified using the comparative $\Delta \mathrm{Ct}$ method $(2-\Delta \Delta \mathrm{CT})$, with tubulin as the reference gene. ${ }^{18}$

\section{Immunoblot analysis}

The muscles were homogenized in Tris-EDTA buffer with a cocktail of protease inhibitors and $1 \mathrm{mM}$ phenylmethylsulfonyl fluoride (PMSF). Proteins were subjected to sodium dodecyl sulphate-polyacrylamide gel electrophoresis (SDS-PAGE), transferred on to polyvinylidene difluoride (PDVF) membranes (EMD Millipore Corp.), and probed with mouse anti-myosin heavy chain (MHC) $(1: 1,000)$ (MF-20, Developmental Studies Hybridoma Bank of the University of Iowa, USA) and mouse anti-tubulin (1:5,000) (Santa Cruz Biotechnology, TX, USA). All immunoreactions were visualized by enhanced chemiluminescence (Thermo Fisher Scientific, Waltham, MA, USA). Images were acquired using Fotodyne FOTO/Analyst Luminary Workstations Systems (Fotodyne, Inc., Walnut Ridge, WI, USA).

\section{Statistics}

For statistical analysis, the one- or two-way analysis of variance with a post hoc Bonferroni multiple-comparison test (Prism $^{\circledR}$, GraphPad Software, San Diego, CA, USA) was utilized. A difference was considered statistically significant at $P<0.05$.

\section{Results \\ MD showed that the PAMAM-OH dendrimer cavities could encapsulate two Ang-(I-7) peptides}

Through MD simulations, the center of mass (COM) distance between three Ang-(1-7) and PAMAM-OH dendrimer was analyzed, which is a way to evaluate the formation of a complex between two molecules or, in this case, the degree of Ang-(1-7) penetration inside the dendrimer cavities. Therefore, COM distance analyses were performed to evaluate the stability of the Ang-(1-7) in the inner cavities of the dendrimer, with particular consideration to the COM of each peptide, along the whole MD trajectory (Figure 1A). Two Ang-(1-7) remained stable from a distance of $10 \AA$ from the COM of the dendrimer during $\sim 60$ ns of molecular
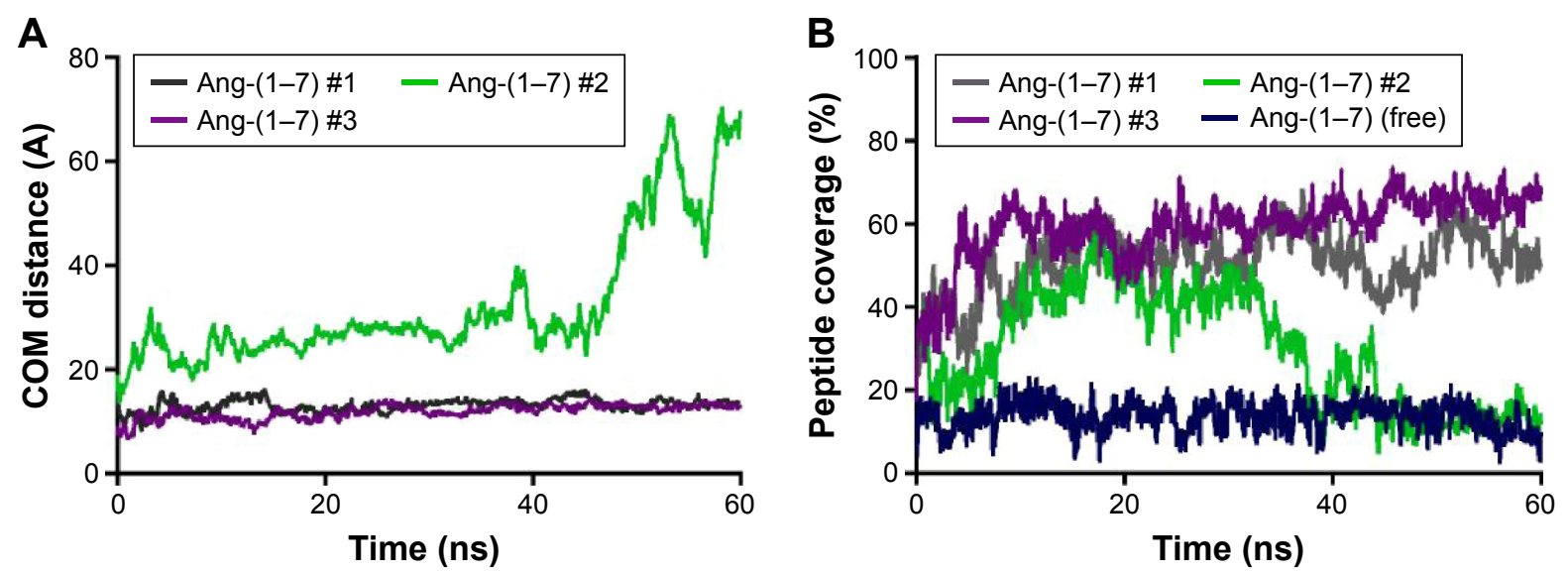

Figure I Neutral PAMAM-OH dendrimer encapsulated two dendrimers and protected them from the environment.

Notes: (A) Comparison of the distance between the COM of Ang-(I-7) peptides and the dendrimer throughout the 60 ns simulation. (B) Percent coverage of Ang-(I-7) in the final $30 \mathrm{~ns}$ of molecular simulation determined by SASA method. The Ang-(I-7) peptides that interacted with the dendrimer are represented in gray, green, and purple. The free Ang- $(\mathrm{I}-7)$ is blue. The "\#" symbol is for distinguishing among the peptides of each system, the "free" peptide being a control system where no dendrimer is involved.

Abbreviations: PAMAM-OH, hydroxyl-terminated poly(amidoamine); COM, center of mass; Ang-(I-7), angiotensin (I-7); SASA, solvent-accessible surface area. 

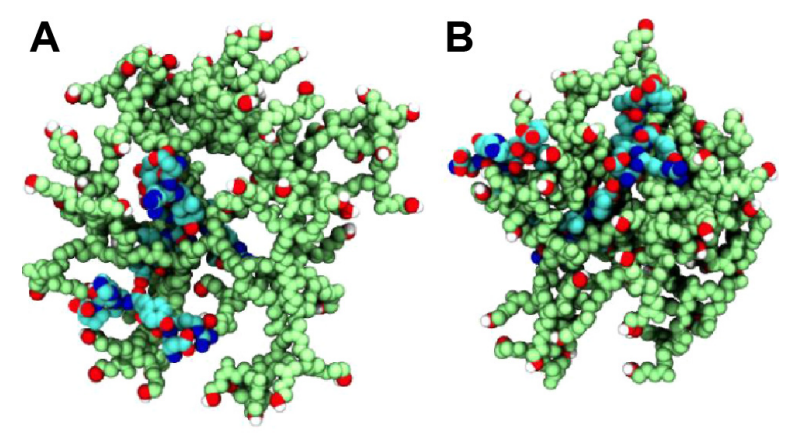

C
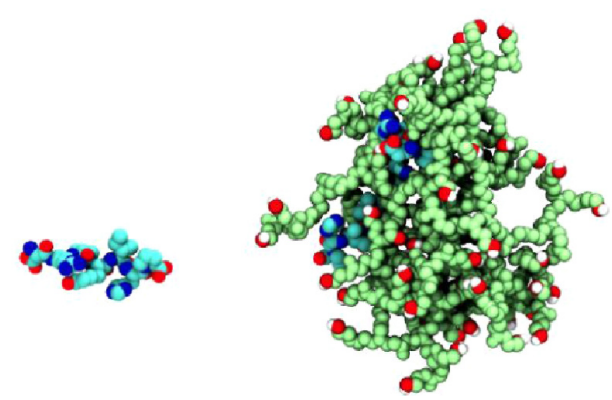

Figure 2 Two Ang-(I-7) peptides remained in the PAMAM-OH cavities. Notes: Snapshots of the peptides/dendrimer complex at (A) $0 \mathrm{~ns}$, (B) $30 \mathrm{~ns}$, and (C) $60 \mathrm{~ns}$ of simulation represent the dendrimer shape and the location of Ang-(I-7) in the cavities or dendrimer surface. The peptides are represented by gray, cyan, and purple spheres, and the PAMAM-OH dendrimer is represented in violet. Abbreviations: Ang-(I-7), angiotensin (I-7); PAMAM-OH, hydroxyl-terminated poly(amidoamine).

simulation, but the third peptide remained $20 \AA$ away from the dendrimer during the initial $30 \mathrm{~ns}$ of simulation time and then escaped from the dendrimer cavities, reaching a distance of $\sim 70 \AA$. The different stages of the Ang-(1-7)/dendrimer complex are depicted in Figure 2.

A

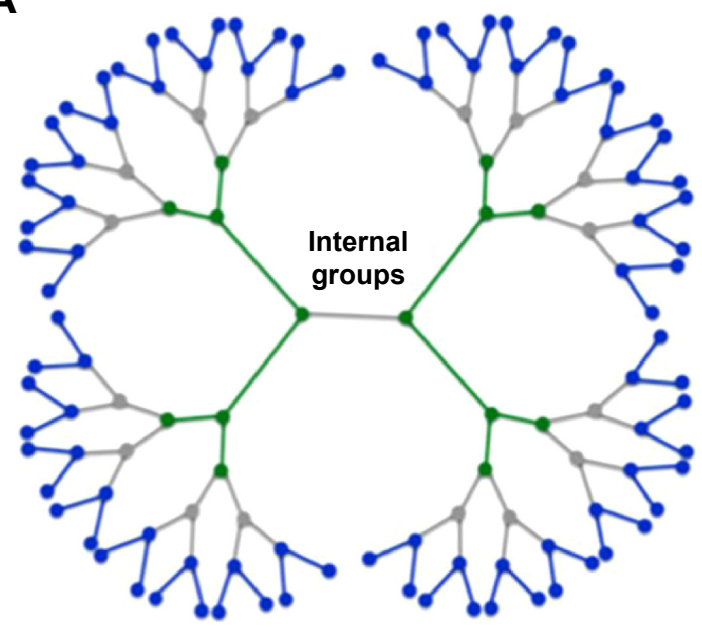

Terminal groups
To test the binding and environmental protection capabilities of the dendrimer, the percentage of peptide solvent coverage was obtained through solvent-accessible surface area (SASA) calculations, which considered only the final $30 \mathrm{~ns}$ of the MD trajectory (Figure 1B). The molecular system of the Ang-(1-7) alone in solution was used as an SASA reference, representing a molecule that is $100 \%$ solvent accessible. Considering this information, one of the Ang-(1-7) - Ang(1-7) \#2 in Figure 1B - that escaped from the dendrimer displayed $<10 \%$ coverage. This was similar to the Ang-(1-7) alone, while the other two peptides showed a dendrimer coverage of $\sim 55 \%$ and $\sim 65 \%$. These analyses indicate that a neutral PAMAM-OH dendrimer could simultaneously encapsulate two Ang-(1-7) in a stable way over the simulation time.

The number of contacts between both molecules was calculated to identify what part of the dendrimer interacted with peptides. Two different dendrimer regions were analyzed, namely, internal cavities and terminal groups, to measure the prevalence of the contacts with peptide amino acids within a cutoff of $3.0 \AA$. Only the side chains of amino acids and the internal and terminal groups in dendrimers were considered (Figure 3A). According to these results, each amino acid, except the Arg2 residue, interacted preferably with internal dendrimer groups rather than with the terminal groups (Figure 3B). As expected, arginine residue was more exposed to solvent. Additionally, hydrophobic amino acids displayed a favorable interaction with internal dendrimer groups. In fact, the internal cavities of neutral dendrimers have been shown to be of hydrophobic nature. ${ }^{34}$

B

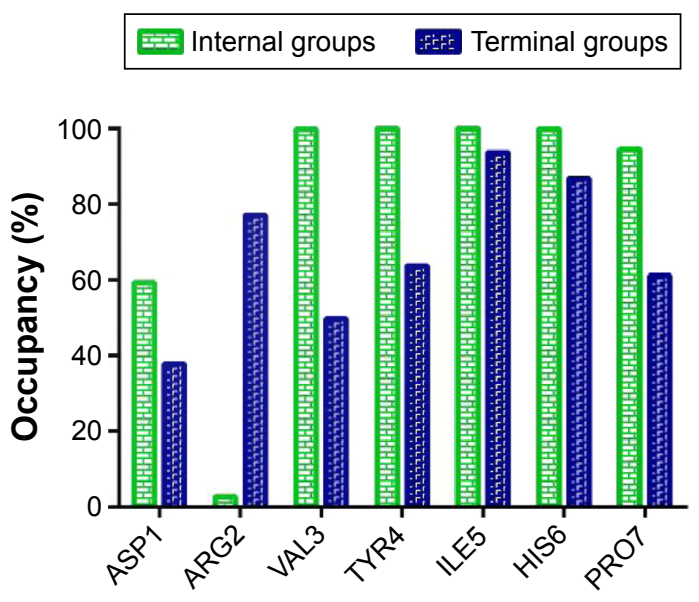

Figure 3 Ang-(I-7) peptides interacted preferentially with the PAMAM-OH dendrimer internal groups.

Notes: (A) Representation of the dendrimer regions considered in this study. Each region is highlighted with different colors. (B) The occupancy percentage of the amino acids in the final $30 \mathrm{~ns}$ of MD simulation. Occupancy was determined by considering the contacts per amino acids at $3.0 \AA$ of distance to the PAMAM-OH dendrimer terminal groups (blue bars) and to the dendrimer internal groups (green bars). Occupancy denotes the average percentage of the two Ang-(I-7) that remained stable within the dendrimer. Abbreviations: Ang-(I-7), angiotensin (I-7); PAMAM-OH, hydroxyl-terminated poly(amidoamine); MD, molecular dynamics. 

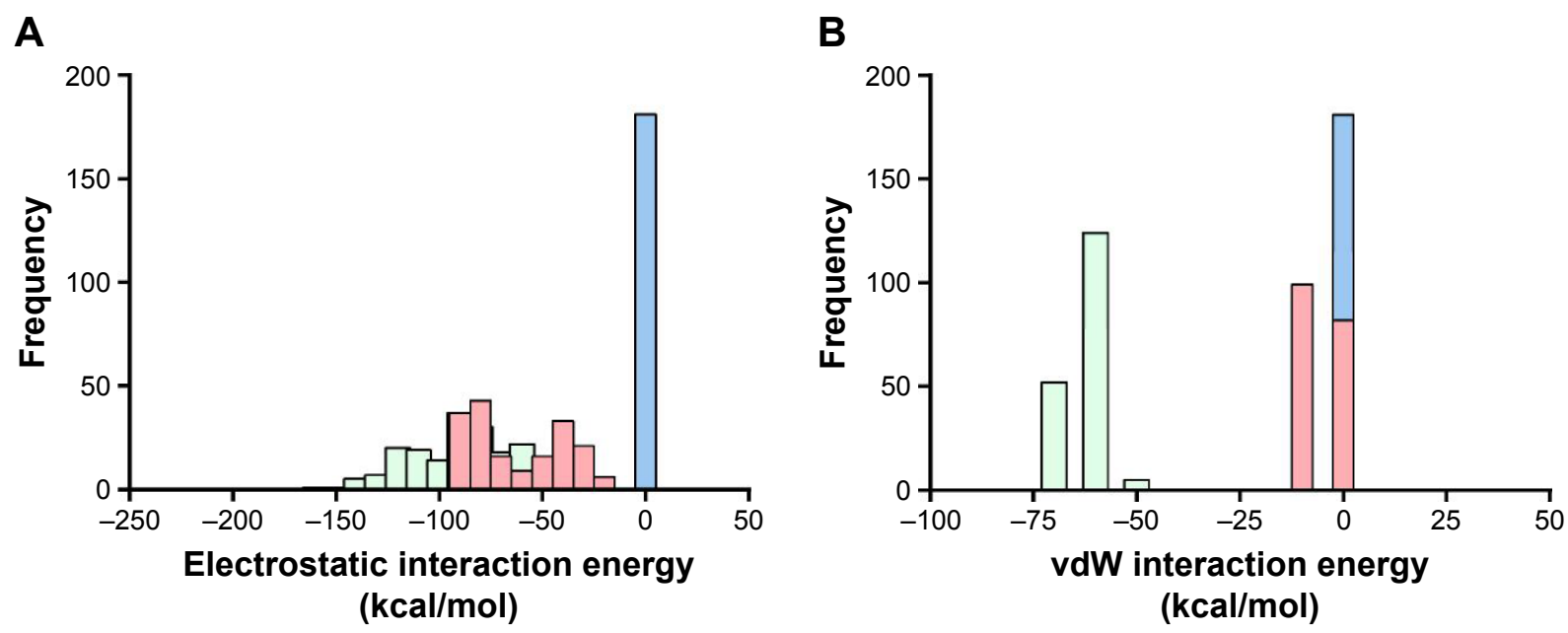

$\square$ Ang-(1-7)/dendrimer $\square$ Dendrimer/free $\square$ Ang-(1-7)/Ang-(1-7)

Figure 4 Electrostatic energy predominated in the Ang-(I-7)/dendrimer interaction.

Notes: Frequency plot of the interaction energy between Ang-(I-7)/dendrimer (green), Ang-(I-7) free/dendrimer (blue), and Ang-(I-7)/Ang-(I-7) (pink), in the final 30 ns of MD simulation. (A) Electrostatic energy, (B) vdW energy. Interaction energy represents an average value for the two peptides that remained stable within the dendrimer. Abbreviations: Ang-(I-7), angiotensin (I-7); MD, molecular dynamics; vdW, van der Waals.

\section{Electrostatic energy drove the interaction between Ang-( $1-7)$ and dendrimers}

Alternatively, electrostatic and vdW interaction energies between Ang-(1-7)/dendrimer, Ang-(1-7) free/dendrimer, and Ang-(1-7)-Ang-(1-7) were calculated during 60 ns of MD simulation. Considering the nature of the interactions driving complex formation, electrostatic energy played a relevant contribution in the three interactions (Figure 4A). This result suggests that the interactions of charged and polar amino acids with the dendrimer are primarily driving complex formation. Though less relevant, vdW interactions are also important in the Ang-(1-7)/dendrimer complex formation, especially due to hydrophobic residues (Ile, Pro, Val), as discussed earlier (Figure 4B). In summary, both peptide/peptide and peptide/ dendrimer interactions displayed high electrostatic interaction energy values, suggesting that both interactions were collaborating in complex formation. Interestingly, despite the lack of charged terminal groups, PAMAM-OH dendrimers could nevertheless establish electrostatic and hydrogen bond interactions with the peptide (Table S2). This interaction can be improved depending on the peptide sequence, which could promote other applications of this neutral, non-toxic dendrimer.

\section{Electrophoretic shift assay revealed that PAMAM-OH formed a complex with two Ang-(I-7) molecules}

To corroborate that the dendrimer was able to encapsulate two peptides utilizing experimental techniques, the complex formation between PAMAM-OH and Ang-(1-7) peptides was followed by an electrophoretic shift assay where PAMAM-OH and Ang-(1-7) peptides were mixed at different molar ratios (dendrimer: Ang-(1-7)). The titration results (Figure 5) demonstrate that the peptide migration patterns differ depending on the respective dendrimer concentration. Additionally, the dendrimer fully retarded peptide mobility in gels (Figure 5, lane 5). These results demonstrate the ability of PAMAM-OH to form complexes with Ang-(1-7), suggesting that one dendrimer molecule

\section{Dendrimer:Ang-(1-7) (molar ratios)}

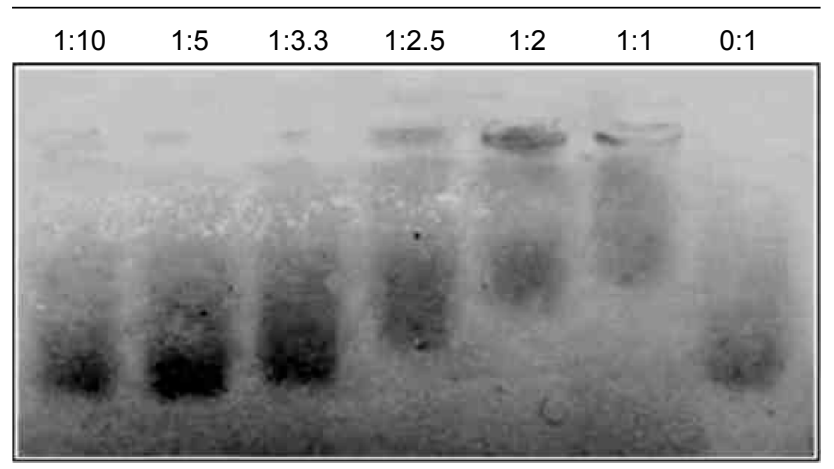

Figure 5 Analysis of the formation of Ang-(I-7)/dendrimer complexes at different dendrimer molar ratios.

Notes: Complex formation was assayed by electrophoretic mobility shift determination in $5 \%$ agarose gel at a $\mathrm{pH}$ of 7.4. The migration of the dendrimer/Ang-(I-7) complexes was visualized by Coomassie staining. The complexes were formed at the following dendrimer/ Ang-(I-7) molar ratios I:I0 (lane I); I:5 (lane 2); I:3.3 (lane 3); I:2.5 (lane 4); I:2 (lane 5); and I:I (lane 6). Lanes I-6 show the formation of the dendrimer/Ang-(I-7) complexes, and lane 7 illustrates the migration of the non-complexed Ang-(I-7), respectively. Abbreviation: Ang-(I-7), angiotensin (I-7). 
could encapsulate two peptides. This assumption was supported by PAMAM-OH loading capacities observed through MD simulations of the Ang-(1-7)/dendrimer complexes at different molar ratios.

\section{IP administration of the Ang- $(I-7) /$ PAMAM-OH complex restored the strength in immobilized skeletal muscle}

Disuse-induced muscle atrophy causes functional changes, including a significant decrease in muscle strength in the immobilized limb. First, the effect of IP administration of either Ang-(1-7) alone or complexed with PAMAM-OH on muscle strength was evaluated. The force-frequency relationship for the isolated GAST of mice unilaterally immobilized during $14 \mathrm{~d}$ and treated with Ang-(1-7) alone or complexed with PAMAM-OH is displayed in Figure 6A. This result shows that IP-injected Ang-(1-7) complexed with PAMAM-OH dendrimer is able to recover the force in immobilized GAST to similar levels of non-immobilized, but not for an IP injection of Ang-(1-7) alone. Maximal tetanic force was also calculated, and results are depicted in Figure 6B. Immobilization induced a muscle strength decrease of $32.6 \% \pm 5.2 \%$ that was prevented when the Ang-(1-7)/PAMAM-OH complex was administered via IP injection. An IP injection of Ang-(1-7) alone was unable to recover muscle strength (Non-Imm Vehicle: 100\% $\pm 9.7 \%$; Imm Vehicle: $67.32 \% \pm 5.2 \%$; Imm Ang-(1-7): 64.7\% 0 3.3\%;
Imm Ang-(1-7)/PAMAM-OH: 97.1\% $0.3 \%$ ). These results contrasted with the recovery of muscle strength induced by Ang-(1-7) alone when systemically delivered by osmotic pumps (Figure S2). The Ang-(1-7)/PAMAM-OH complex administered by osmotic pumps had an effect similar to Ang-(1-7) alone in immobilized muscle and reached muscle strength values similar to those of non-immobilized muscle (Non-Imm Vehicle: 100\% $\pm 6.2 \%$; Imm Vehicle: 61.4\% 77.2\%; Imm Ang-(1-7): 97.3\% 44.8\%; Imm Ang(1-7)/PAMAM-OH: 98.1\% $\pm 5.0 \%$ ).

These results indicate that IP administered Ang-(1-7) could restore muscle strength only when forming a complex with PAMAM-OH, suggesting the possible protection of Ang-(1-7) by PAMAM-OH.

\section{Ang-(I-7)/PAMAM-OH complex, IP administered, recovered the fiber diameter in immobilized muscle}

The muscle fiber diameter, which characteristically declines in cases of muscle atrophy by disuse, ${ }^{17}$ was evaluated. To accomplish this, WGA staining in transversal sections of the GAST muscle was performed. Immobilized muscle displayed an important decrease in fiber diameter (Figure 7Aa versus $\mathrm{Ab}$ ), while IP administration of Ang(1-7) only recovered the fiber diameter when complexed with PAMAM-OH (Figure 7Ag versus $\mathrm{Ah}$ ) and not when was administered alone (Figure 7 Ac versus Ad).

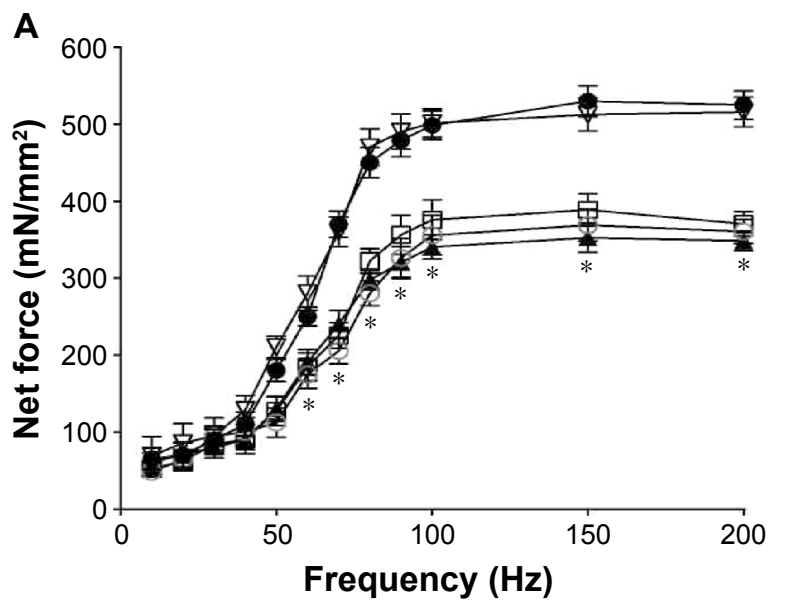

- Control Non-Imm $\odot$ Imm-Vehicle Đ Imm-Ang-(1-7)
\& Imm-PAMAM-OH Imm-Ang-(1-7)/PAMAM-OH
B

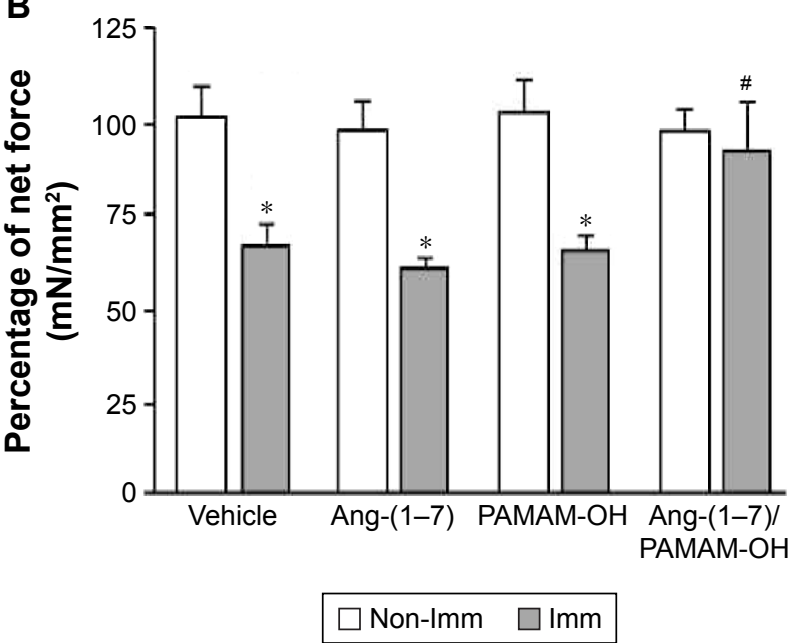

Figure 6 IP administrated Ang-(I-7)/PAMAM-OH complex prevented the decrease in isometric force of disused muscle.

Notes: Non-immobilized (Non-Imm) or contralateral immobilized for $14 \mathrm{~d}$ (Imm) GAST muscles from mice intraperitoneally injected with the vehicle, Ang-( I-7), PAMAM-OH, or Ang-(I-7)/PAMAM-OH complex were excised. (A) Curve of force versus frequency was determined. (B) Maximal isometric strength ( $\left.\mathrm{mN} / \mathrm{mm}^{2}\right)$ was evaluated. Values represent the mean \pm SD of triplicate independent experiments. In each experiment, five to seven mice were used for each experimental condition. ( $* P<0.05$ versus vehicletreated/non-immobilized GAST; ${ }^{\# P}<0.05$ versus vehicle-treated/immobilized GAST.)

Abbreviations: IP, intraperitoneal; Ang-(I-7), angiotensin (I-7); PAMAM-OH, hydroxyl-terminated poly(amidoamine); GAST, gastrocnemius; SD, standard deviation. 
A
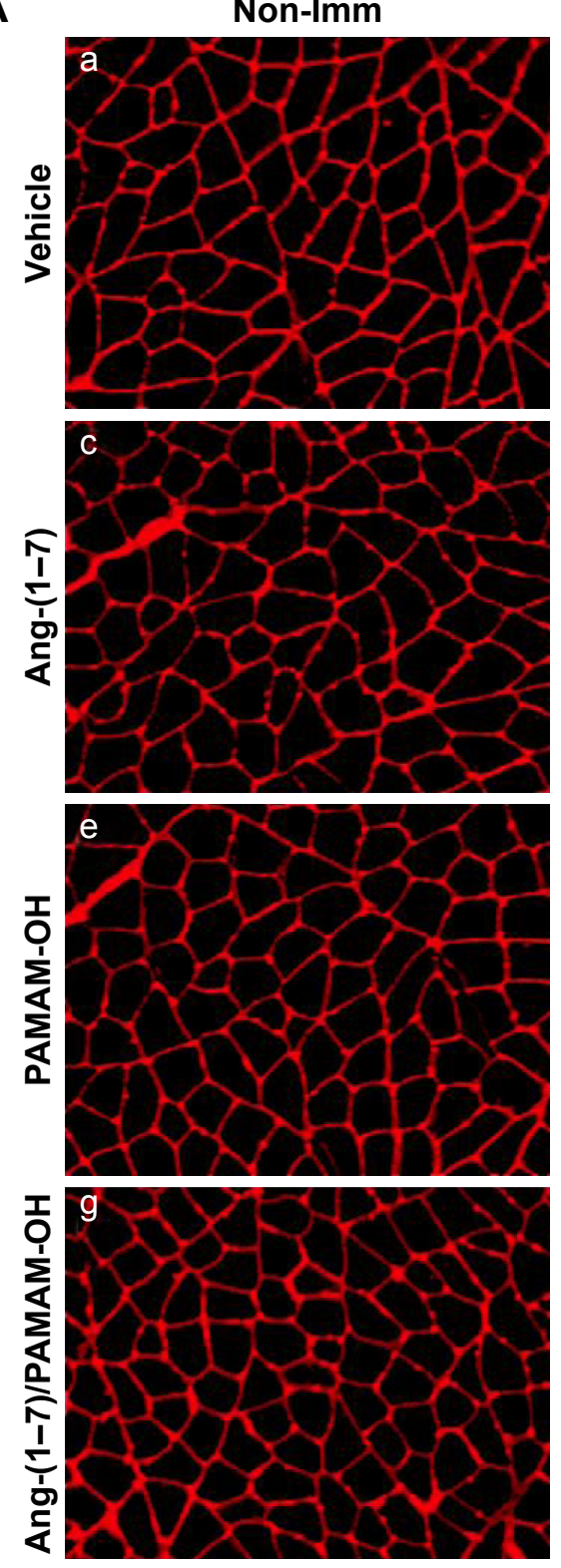

Imm
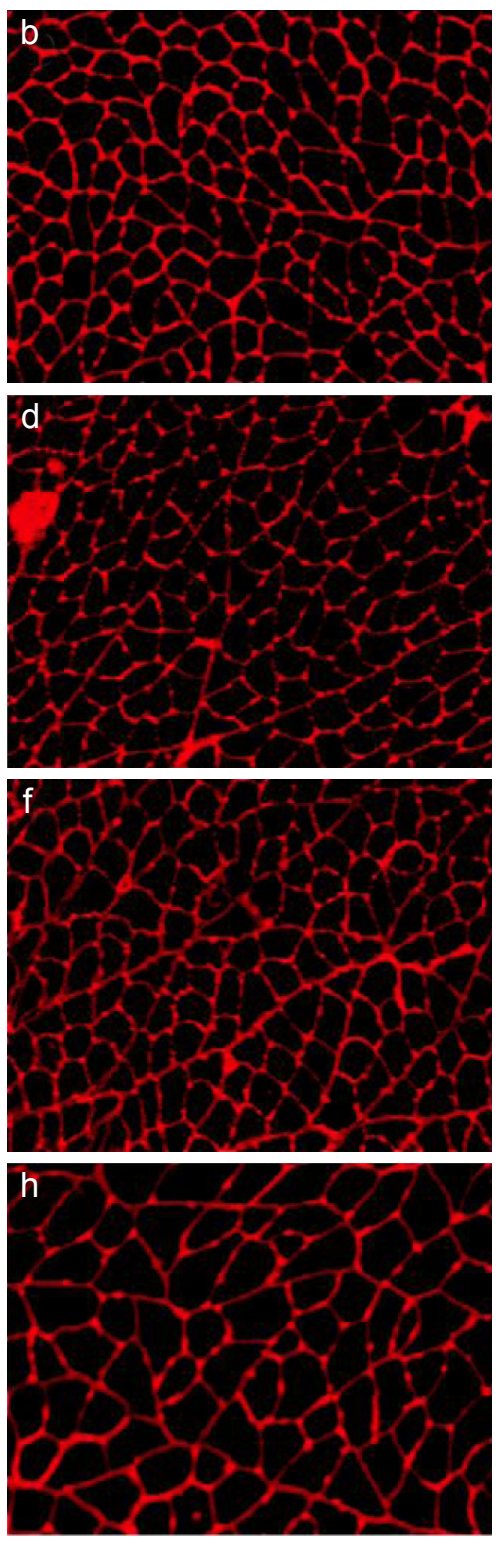

B

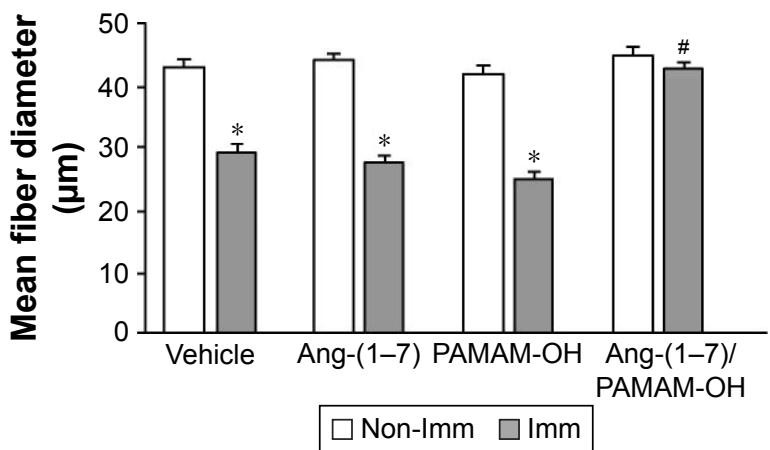

Figure 7 IP injection of Ang-(I-7)/PAMAM-OH complex prevented the decreased muscle fiber diameter under disuse.

Notes: (A) The fiber diameter of non-immobilized (Non-Imm; a, c, e, g) or contralateral immobilized for I4 d (Imm; b, d, f, h) GAST from mice IP injected with the vehicle (a, b), Ang-(I-7) (c, d), PAMAM-OH (e, f), or Ang-(I-7)/PAMAM-OH (g, h) complex were compared. Muscle cross-sections were stained with WGA to delimit muscle fiber sarcolemma. (B) Minimal Feret diameters were determined in GAST cross-sections from (A). The values are expressed as the mean fiber diameter. The quantification is representative of triplicate independent experiments. Each experiment used five to seven mice for each experimental condition. Values correspond to the mean \pm SD. $\left(* P<0.05\right.$ versus vehicle-treated/non-immobilized GAST; ${ }^{*} P<0.05$ versus vehicle-treated/immobilized GAST.)

Abbreviations: IP, intraperitoneal; Ang-(I-7), angiotensin (I-7); PAMAM-OH, hydroxyl-terminated poly(amidoamine); GAST, gastrocnemius; WGA, wheat germ agglutinin; $\mathrm{SD}$, standard deviation. 
A

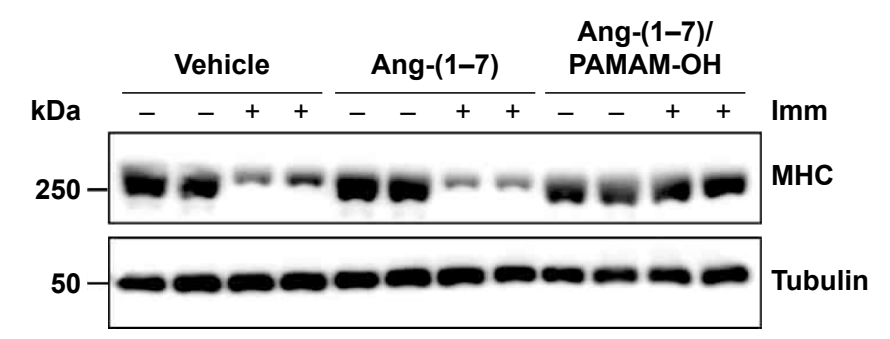

C

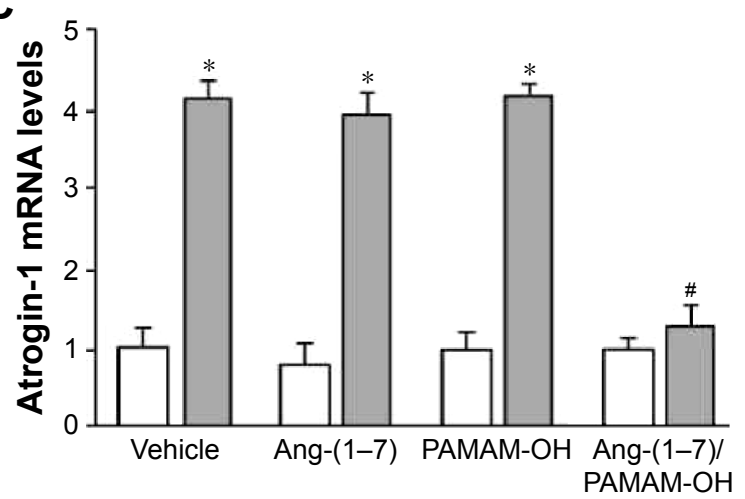

B

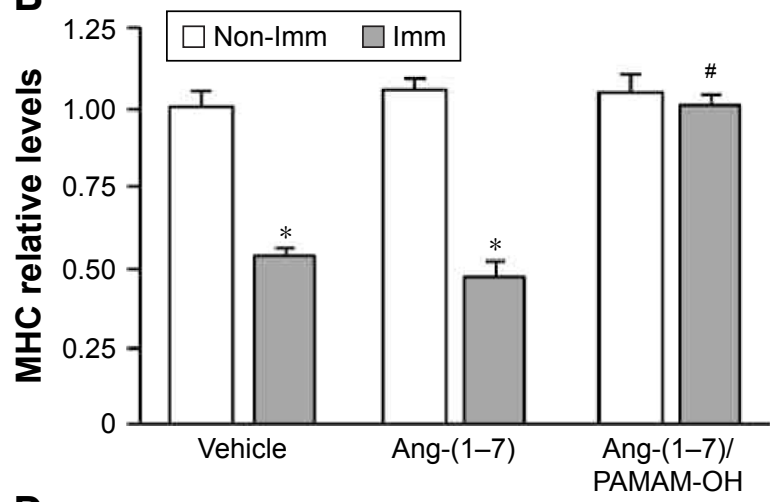

D

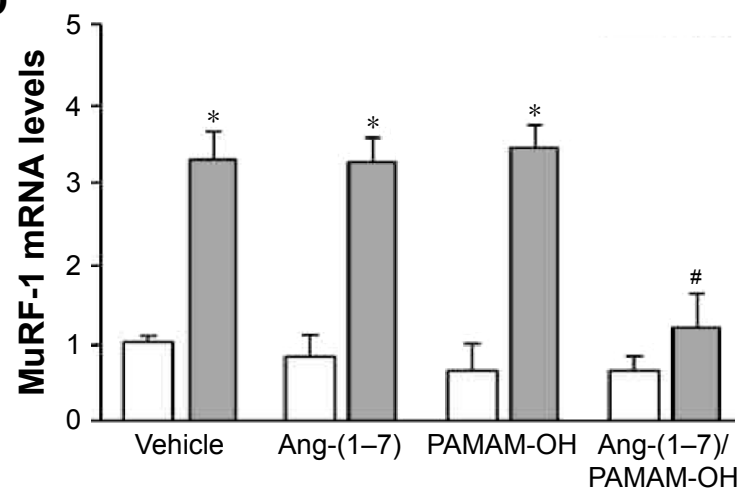

$\square$ Non-Imm $\square \mathrm{Imm}$

Figure 8 Ang-(I-7)/PAMAM-OH complex intraperitoneally administrated prevented the decreased MHC levels and increased E3 ligases of disused muscle. Notes: The hind limb of mice intraperitoneally injected with vehicle,Ang-(I-7), or Ang-(I-7)/PAMAM-OH complex was unilaterally immobilized for I4 d. (A) The immobilized $(\mathrm{Imm})$ and non-immobilized (Non-Imm) GAST were isolated and homogenized to evaluate MHC protein levels through Western blot analysis. Tubulin levels were used as the loading control. Molecular weight markers are shown in kilodaltons. (B) Quantitative analysis of the experiments from (A). The levels of MHC normalized to tubulin are expressed relative to vehicle-injected/non-immobilized GAST and correspond to the mean \pm SD from triplicate independent experiments. In each experiment, five to seven mice were used for each experimental condition. Detection of atrogin-I (C) and MuRF-I (D) mRNA levels through RT-qPCR used tubulin as the reference gene. Expressions are shown as the fold of induction relative to vehicle-treated/non-immobilized GAST and the values corresponding to the mean \pm SD of triplicate independent experiments. In each experiment, five to seven mice were used for each experimental condition $\left(* P<0.05\right.$ versus vehicle-treated/non-immobilized GAST; ${ }^{*}<<0.05$ versus vehicle-treated/ immobilized GAST.)

Abbreviations: Ang-(I-7), angiotensin (I-7); PAMAM-OH, hydroxyl-terminated poly(amidoamine); MHC, myosin heavy chain; GAST, gastrocnemius; SD, standard deviation; mRNA, messenger RNA; RT-qPCR, reverse transcription quantitative real-time polymerase chain reaction.

The PAMAM-OH alone has no effect on the decrease of fiber diameter (Figure 7Ae versus Af). The quantification of mean Feret diameters from Figure $7 \mathrm{~A}$ is depicted in Figure 7B (Non-Imm Vehicle: $42.6 \pm 0.8 \mu \mathrm{m}$; Imm Vehicle: 26.5 $\pm 1.2 \mu \mathrm{m}$; Imm Ang-(1-7): $25.4 \pm 0.9 \mu \mathrm{m}$; Imm Ang-(1-7)/ PAMAM-OH: $41.7 \pm 1.1 \mu \mathrm{m})$. These results contrasted with the administration of Ang-(1-7) by osmotic pump, which prevented the decrease in fiber diameter with both Ang-(1-7) alone and when complexed with PAMAM-OH (Figure S3A). In addition, the results presented the quantification of fiber diameter from the data (Figure S3B).

These results indicate that the Ang-(1-7)/PAMAM-OH complex was more capable of preventing the decrease in muscle fiber diameter than Ang-(1-7) alone when administrated by IP injection in disuse-induced muscle atrophy.

\section{IP administration of Ang-( I-7)/PAMAM- $\mathrm{OH}$ complex prevented the decrease of $\mathrm{MHC}$ and the increase of atrogin-I and MuRF-I expression in immobilized skeletal muscle}

A principal sarcomeric protein downregulated in skeletal muscle atrophy is MHC. The effect of IP injection of Ang-(1-7) alone or complexed with PAMAM-OH on the MHC levels was assayed during immobilization. The levels of MHC were downregulated in immobilized GAST by $\sim 47 \%$ compared to non-immobilized muscle (Figure 8A and B). Remarkably, only the Ang-(1-7)/PAMAM-OH complex, but not Ang-(1-7) alone, inhibited the decrease in MHC levels induced by immobilization when administered by IP injection (Non-Imm-Vehicle: 1.00 \pm 0.08 ; Imm Vehicle: 


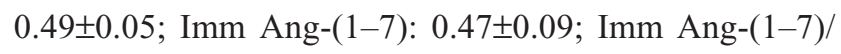
PAMAM-OH: 1.01 \pm 0.04 ) (Figure $8 \mathrm{~A}$ and $\mathrm{B}$ ). In accordance with our previous results, both Ang-(1-7) alone and the Ang-(1-7)/PAMAM-OH complex inhibited the decrease in $\mathrm{MHC}$ levels induced by disuse when administered by osmotic pumps (Figure 4A and B).

Furthermore, the effect of Ang-(1-7) alone or complexed with PAMAM-OH was evaluated on the expression of two UPP markers upregulated by immobilization: atrogin-1 (4.17 \pm 0.22 fold of induction) (Figure 8C) and MuRF-1 (3.33 \pm 0.35 fold of induction) (Figure $8 \mathrm{D})$. The IP administration of the Ang-(1-7)/PAMAM-OH complex completely prevented the immobilization-induced upregulation of atrogin-1 (1.29 \pm 0.25 fold of induction) (Figure 8C) and MuRF-1 (1.21 \pm 0.42 fold of induction) (Figure 8D). These effects were lost when Ang-(1-7) alone was IP injected (fold of induction: $3.96 \pm 0.27$ for atrogin-1; $3.29 \pm 0.32$ for MuRF-1). In contrast, both Ang-(1-7) and the Ang-(1-7)/ PAMAM-OH complex administered by osmotic pumps prevented the upregulation of atrogin-1 (Figure S4C) and MuRF-1 expression (Figure S4D).

These results indicate that the Ang-(1-7)/PAMAM-OH complex was more capable of preventing the decrease of MHC levels and the upregulation of atrogin-1 and MuRF-1 than Ang-(1-7) when administrated by IP injection in disuseinduced muscle atrophy.

\section{Discussion}

The biological effects of Ang-(1-7) are widely described in the heart, kidney, nervous system, liver, and skeletal muscle, among other tissues. ${ }^{35,36}$ Due to the short half-life of Ang-(1-7), studies evaluating this peptide regularly use osmotic pumps for administration to ensure continuous peptide delivery and release. In this context, designing new delivery methods that increase Ang-(1-7) bioavailability is critical for therapeutic use. To date, only an orally administered formulation of Ang-(1-7) and cyclodextrin is effective in preventing the intestinal degradation of the peptide. However, there are no reports of injectable formulations that delay rapid Ang-(1-7) degradation in the body. This study found a new formulation, based on the Ang-(1-7) complex with the PAMAM-OH dendrimer, which is able to exert an anti-atrophic effect when IP injected in a disuse-induced muscle atrophy model.

Here, we have shown by MD that the dendrimer is able to establish both electrostatic and vdW interactions with Ang-(1-7) peptides - with the first one being the most relevant - mainly due to H-bonds. PAMAM-OH dendrimer, due to its non-charged nature, has been shown to have a lower diameter due to internal H-bonds and hydrophobic interactions - differently to other charged dendrimers such as PAMAM- $\mathrm{NH}_{2}-$ which, due to its charge-charge repulsion, increases its diameter. This effect is contributing to make PAMAM-OH cavities into hydrophobic ones, decreasing the penetration of waters to the inner cavities. On the other hand, inner cavities of PAMAM-OH dendrimers also possess amide groups that can establish hydrogen bonds with the peptides.

This phenomenon was corroborated using an electrophoretic mobility shift assay, which showed that the dendrimer fully retarded peptide mobility at a molar ratio of $2: 1$, as observed in the MD simulations. In total, these findings suggest that PAMAM-OH is a reasonable alternative for the protection of peptides, the half-life of which, in a biological environment, may be shorter in a solution than in a complex with this dendrimer. Furthermore, the peptide interactions were also dependent on the amino acid sequence. Therefore, improvements on peptide/dendrimer affinity could be formulated using dendrimers decorated with mixtures of neutral (hydroxyl) and charged groups (amine, carboxylate, etc.), as previously suggested. ${ }^{37}$

The anti-atrophic effects of Ang-(1-7) on three typical features of disuse-induced muscle atrophy were recently described, specifically with regard to recovering muscle strength and protecting against the loss of myofibrillar proteins and UPP activation. Interestingly, these effects were reported when Ang-(1-7) was administered through osmotic pumps. This study IP injected Ang-(1-7) alone or complexed with the dendrimer PAMAM-OH, finding that only the Ang(1-7)/PAMAM-OH complex maintained the anti-atrophic effects of Ang-(1-7), while Ang-(1-7) IP injections displayed no improvement in muscle atrophy parameters. This suggests that Ang-(1-7) requires protection when injected. Moreover, the effects of IP injections with the Ang-(1-7)/ PAMAM-OH complex were comparable to the effects of Ang-(1-7) administrated by osmotic pumps.

Advances in muscle atrophy therapy are currently focused on administering amino acids or on exercises. However, there are no described molecules or peptides that affect many types of muscle atrophy. In this context, Ang-(1-7) has a demonstrated anti-atrophic effect on Ang-II-dependent and -independent models, such as in disuse, sepsis, or Ang-II-induced cachexia. ${ }^{11,15-18}$ This study is highly relevant for muscle biology, particularly with regard to pathological statuses that induce muscle atrophy, such as sepsis, that cannot be treated with oral drug therapies or exercise. Therefore, further studies should be conducted to assess the therapeutic 
effects of an injectable Ang-(1-7)/PAMAM-OH complex on sepsis-induced muscle wasting.

The presently reported findings could be biologically important in the treatment of numerous chronic and highly prevalent diseases, including cardiovascular, renal, and inflammatory conditions, as well as diabetes, cancer, and glaucoma. ${ }^{36}$ The effect of Ang-(1-7) has been demonstrated in cardiovascular pathologies such as cardiac remodeling, fibrosis, hypertension, and endothelial dysfunction. ${ }^{36,38}$ In the case of plasma glucose handling, target tissues evidence beneficial effects of Ang-(1-7), including in skeletal muscle. ${ }^{35}$ Furthermore, several kidney conditions can be improved by Ang-(1-7) treatment, especially those involving fibrosis. ${ }^{39}$ Therefore, novel treatments against several chronic diseases that utilize Ang-(1-7) as a principal molecule are also potentially beneficial therapeutic strategies against induced skeletal muscle atrophy.

\section{Conclusion}

We determined the feasibility of using neutral dendrimers as therapeutic peptide carriers. This study was based on the biological activity of Ang-(1-7) on skeletal muscle atrophy, and it demonstrated that a novel formulation of Ang-(1-7) with a dendrimer could improve the anti-atrophic properties of Ang-(1-7) when IP injected. Therefore, the Ang-(1-7)/ dendrimer complex could serve as a protective delivery system that improves the biological functions of the peptide in vivo. Non-toxic effects were observed in in vivo assays using Ang-(1-7)/PAMAM-OH. At the same time, this study is a contribution to the design of novel dendrimers decorated with neutral (hydroxyl) and/or charged groups (amine, carboxylate, etc.) with the aim of improving or modulating the half-life or kinetic release of peptides and therefore improving the biological effect of peptides, as was demonstrated with Ang-(1-7) in this study.

\section{Acknowledgments}

This study was supported by research grants from Association-Francaise Contre Les Myopathies AFM 16670 (CCV); FONDECYT 1120380, 1161646 (CCV), 1161288 (FS); Millennium Institute on Immunology and Immunotherapy P09-016-F (CCV, FS), UNAB DI-741-15/N (CCV, FS). VMM, JA, and JCR thank Conicyt for a PhD Scholarship. VMM. thanks CONICYT + PAI/“CConcurso Nacional Tesis de Doctorado en la Empresa” 2014 (781413007). FGN, VMM, and IAD thank Fraunhofer Chile Research, InnovaChile CORFO (FCR-CSB 09CEII-6991), Anillo Científico ACT1107, and RED CYTED 214RT0482 for their support.
The Centro Interdisciplinario de Neurociencia de Valparaíso (CINV) is a Millennium Institute supported by the Millennium Scientific Initiative of the Ministerio de Economía, Fomento y Turismo.

\section{Disclosure}

The authors report no conflicts of interest in this work.

\section{References}

1. Avila-Salas F, Sandoval C, Caballero J, et al. Study of interaction energies between the PAMAM dendrimer and nonsteroidal anti-inflammatory drug using a distributed computational strategy and experimental analysis by ESI-MS/MS. J Phys Chem B. 2012;116(7):2031-2039.

2. Vergara-Jaque A, Comer J, Monsalve L, Gonzalez-Nilo FD, Sandoval C. Computationally efficient methodology for atomic-level characterization of dendrimer-drug complexes: a comparison of amine- and acetylterminated PAMAM. J Phys Chem B. 2013;117(22):6801-6813.

3. Marquez-Miranda V, Camarada MB, Araya-Duran I, Varas-Concha I, Almonacid DE, Gonzalez-Nilo FD. Biomimetics: from bioinformatics to rational design of dendrimers as gene carriers. PLoS One. 2015; 10(9):e0138392.

4. Dai H, Navath RS, Balakrishnan B, et al. Intrinsic targeting of inflammatory cells in the brain by polyamidoamine dendrimers upon subarachnoid administration. Nanomedicine (Lond). 2010;5(9):1317-1329.

5. Kannan S, Dai H, Navath RS, et al. Dendrimer-based postnatal therapy for neuroinflammation and cerebral palsy in a rabbit model. Sci Transl Med. 2012;4(130):130ra146.

6. Iusuf D, Henning RH, van Gilst WH, Roks AJ. Angiotensin-(1-7): pharmacological properties and pharmacotherapeutic perspectives. Eur J Pharmacol. 2008;585(2-3):303-312.

7. Sandri M. Protein breakdown in muscle wasting: role of autophagylysosome and ubiquitin-proteasome. Int J Biochem Cell Biol. 2013;45(10): 2121-2129.

8. Cabello-Verrugio C, Cordova G, Salas JD. Angiotensin II: role in skeletal muscle atrophy. Curr Protein Pept Sci. 2012;13(6):560-569.

9. Rezk BM, Yoshida T, Semprun-Prieto L, Higashi Y, Sukhanov S, Delafontaine P. Angiotensin II infusion induces marked diaphragmatic skeletal muscle atrophy. PLoS One. 2012;7(1):e30276.

10. Sukhanov S, Semprun-Prieto L, Yoshida T, et al. Angiotensin II, oxidative stress and skeletal muscle wasting. Am J Med Sci. 2011;342(2): $143-147$.

11. Morales MG, Abrigo J, Meneses C, et al. The Ang-(1-7)/Mas-1 axis attenuates the expression and signalling of TGF-beta 1 induced by AngII in mouse skeletal muscle. Clin Sci. 2014;127(4):251-264.

12. Prasannarong M, Santos FR, Henriksen EJ. ANG-(1-7) reduces ANG IIinduced insulin resistance by enhancing Akt phosphorylation via a Mas receptor-dependent mechanism in rat skeletal muscle. Biochem Biophys Res Commun. 2012;426(3):369-373.

13. Xue H, Zhou L, Yuan P, et al. Counteraction between angiotensin II and angiotensin-(1-7) via activating angiotensin type I and Mas receptor on rat renal mesangial cells. Regul Pept. 2012;177(1-3):12-20.

14. Yang HY, Bian YF, Zhang HP, et al. Angiotensin-(1-7) treatment ameliorates angiotensin II-induced HUVEC apoptosis. Clin Exp Pharmacol Physiol. 2012;39(12):1004-1010.

15. Cisternas F, Morales MG, Meneses C, et al. Angiotensin-(1-7) decreases skeletal muscle atrophy induced by angiotensin II through a Mas receptor-dependent mechanism. Clin Sci. 2015;128(5):307-319.

16. Meneses C, Morales MG, Abrigo J, Simon F, Brandan E, CabelloVerrugio C. The angiotensin-(1-7)/Mas axis reduces myonuclear apoptosis during recovery from angiotensin II-induced skeletal muscle atrophy in mice. Pflugers Arch. 2015;467(9):1975-1984.

17. Morales MG, Abrigo J, Acuna MJ, et al. Angiotensin-(1-7) attenuates disuse skeletal muscle atrophy via the Mas receptor. Dis Model Mech. 2016;9(4):441-449. 
18. Morales MG, Olguin H, Di Capua G, Brandan E, Simon F, CabelloVerrugio C. Endotoxin-induced skeletal muscle wasting is prevented by angiotensin-(1-7) through a p38 MAPK-dependent mechanism. Clin Sci. 2015;129(6):461-476.

19. Acuna MJ, Pessina $\mathrm{P}$, Olguin $\mathrm{H}$, et al. Restoration of muscle strength in dystrophic muscle by angiotensin-1-7 through inhibition of TGF-beta signalling. Hum Mol Genet. 2014;23(5):1237-1249.

20. Vanommeslaeghe K, Hatcher E, Acharya C, et al. CHARMM general force field: a force field for drug-like molecules compatible with the CHARMM all-atom additive biological force fields. J Comput Chem. 2010;31(4):671-690.

21. Vanommeslaeghe K, MacKerell AD Jr. Automation of the CHARMM General Force Field (CGenFF) I: bond perception and atom typing. J Chem Inf Model. 2012;52(12):3144-3154.

22. Vanommeslaeghe K, Raman EP, MacKerell AD Jr. Automation of the CHARMM General Force Field (CGenFF) II: assignment of bonded parameters and partial atomic charges. J Chem Inf Model. 2012;52(12): $3155-3168$

23. Humphrey W, Dalke A, Schulten K. VMD: visual molecular dynamics. J Mol Graph. 1996;14(1):33-38, 27-38.

24. Best RB, Zhu X, Shim J, et al. Optimization of the additive CHARMM all-atom protein force field targeting improved sampling of the backbone phi, psi and side-chain chi(1) and chi(2) dihedral angles. JChem Theory Comput. 2012;8(9):3257-3273.

25. Jorgensen WL, Chandrasekhar J, Madura JD, Impey RW, Klein ML. Comparison of simple potential functions for simulating liquid water. J Chem Phys. 1983;79(2):926-935.

26. Phillips JC, Braun R, Wang W, et al. Scalable molecular dynamics with NAMD. J Comput Chem. 2005;26(16):1781-1802.

27. Morris GM, Huey R, Lindstrom W, et al. AutoDock4 and AutoDockTools4: automated docking with selective receptor flexibility. J Comput Chem. 2009;30(16):2785-2791.

28. Martyna GJ, Tobias DJ, Klein ML. Constant pressure molecular dynamics algorithms. J Chem Phys. 1994;101(5):4177-4189.

29. Essmann U, Perera L, Berkowitz ML, Darden T, Lee H, Pedersen LG. A smooth particle mesh Ewald method. J Chem Phys. 1995;103(19): 8577-8593.
30. Abrigo J, Rivera JC, Simon F, Cabrera D, Cabello-Verrugio C. Transforming growth factor type beta (TGF-beta) requires reactive oxygen species to induce skeletal muscle atrophy. Cell Signal. 2016;28(5):366-376.

31. Cabrera D, Gutierrez J, Cabello-Verrugio C, et al. Andrographolide attenuates skeletal muscle dystrophy in mdx mice and increases efficiency of cell therapy by reducing fibrosis. Skeletal Muscle. 2014;4:6.

32. Morales MG, Cabello-Verrugio C, Santander C, Cabrera D, Goldschmeding R, Brandan E. CTGF/CCN-2 over-expression can directly induce features of skeletal muscle dystrophy. J Pathol. 2011;225(4): 490-501.

33. Morales MG, Cabrera D, Cespedes C, et al. Inhibition of the angiotensinconverting enzyme decreases skeletal muscle fibrosis in dystrophic mice by a diminution in the expression and activity of connective tissue growth factor (CTGF/CCN-2). Cell Tissue Res. 2013;353(1): 173-187.

34. Geitner NK, Wang B, Andorfer RE, Ladner DA, Ke PC, Ding F. Structure-function relationship of PAMAM dendrimers as robust oil dispersants. Environ Sci Technol. 2014;48(21):12868-12875.

35. Cabello-Verrugio C, Morales MG, Rivera JC, Cabrera D, Simon F. Renin-angiotensin system: an old player with novel functions in skeletal muscle. Med Res Rev. 2015;35(3):437-463.

36. Passos-Silva DG, Brandan E, Santos RA. Angiotensins as therapeutic targets beyond heart disease. Trends Pharmacol Sci. 2015;36(5): 310-320.

37. Marquez-Miranda V, Penaloza PJ, Araya-Duran I, et al. Effect of terminal groups of dendrimers in the complexation with antisense oligonucleotides and cell uptake. Nanoscale Res Lett. 2016;11(1):1-13.

38. Mendoza-Torres E, Oyarzun A, Mondaca-Ruff D, et al. ACE2 and vasoactive peptides: novel players in cardiovascular/renal remodeling and hypertension. Ther Adv Cardiovasc Dis. 2015;9(4):217-237.

39. Lv LL, Liu BC. Role of non-classical renin-angiotensin system axis in renal fibrosis. Front Physiol. 2015;6:117. 


\section{Supplementary materials}

A

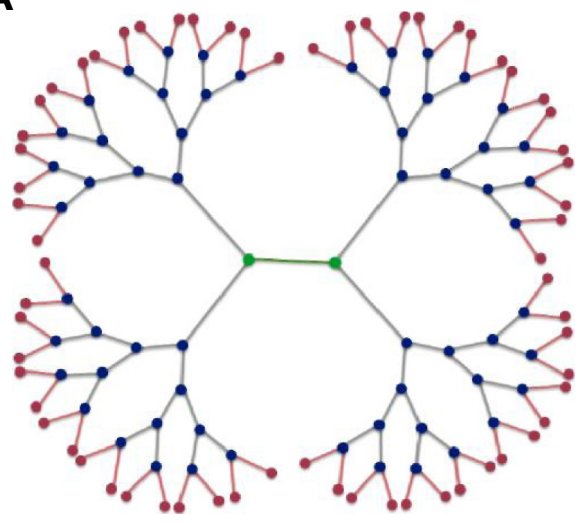

Ethylenediamine core

Internal dendrons (MON)

Terminal hydroxyl groups
B

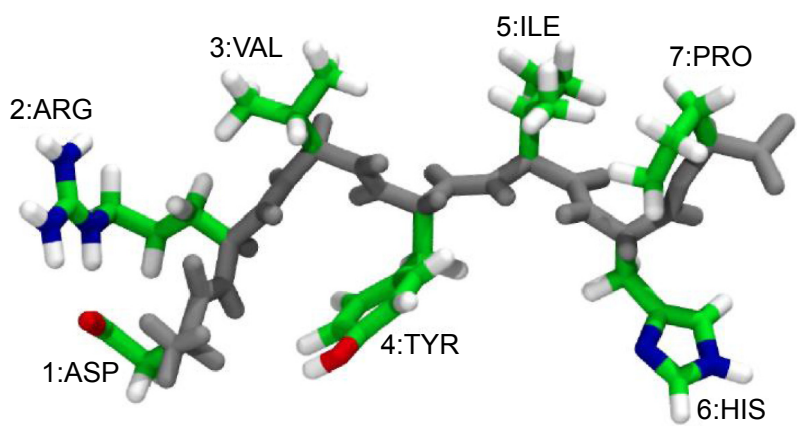

Figure SI Scheme of molecular structures of Ang-(I-7) peptide and PAMAM-OH dendrimer.

Notes: Molecular structures of (A) PAMAM-OH dendrimer generation 4 and (B) Ang-(I-7) peptide.

Abbreviations: Ang-(I-7), angiotensin (I-7); PAMAM-OH, hydroxyl-terminated poly(amidoamine); MON, monomer.

Table SI Details of molecular systems built in this study

\begin{tabular}{llllll}
\hline System & $\begin{array}{l}\text { Total number } \\
\text { of peptides }\end{array}$ & $\begin{array}{l}\text { Number of } \\
\text { water molecules }\end{array}$ & $\begin{array}{l}\text { Number of } \\
\mathbf{C l} \text { ions }\end{array}$ & $\begin{array}{l}\text { Number of } \\
\mathbf{N a}^{+} \text {ions }\end{array}$ & $\begin{array}{l}\text { Total number } \\
\text { of atoms }\end{array}$ \\
\hline Ang-(I-7) alone & 1 & 3.183 & 9 & 9 & 9.693 \\
PAMAM-OH & 0 & 25.672 & 72 & 72 & 79.340 \\
Ang-(I-7)/PAMAM-OH & 3 & 77.272 & 218 & 218 & 234.810 \\
\hline
\end{tabular}

Abbreviations: Ang-(I-7), angiotensin (I-7); PAMAM-OH, hydroxyl-terminated poly(amidoamine).

Table S2 Hydrogen bonds between peptides and PAMAM-OH

\begin{tabular}{lllll}
\hline Peptide & ASPI & TYR4 & ILE5 & HIS6 \\
\hline Peptide \#I & MONI3 (44.8I\%) & MONI2 (52.22\%) & & \\
Peptide \#3 & MON38 (17.04\%) & MONI6 (13.15\%) & MON8 (20.19\%) & MON34 (10\%) \\
\hline
\end{tabular}

Notes: Time occupancy over the last $30 \mathrm{~ns}$ of MD simulation is shown in brackets. MON denotes an internal monomer of the dendrimer.

Abbreviation: PAMAM-OH, hydroxyl-terminated poly(amidoamine).

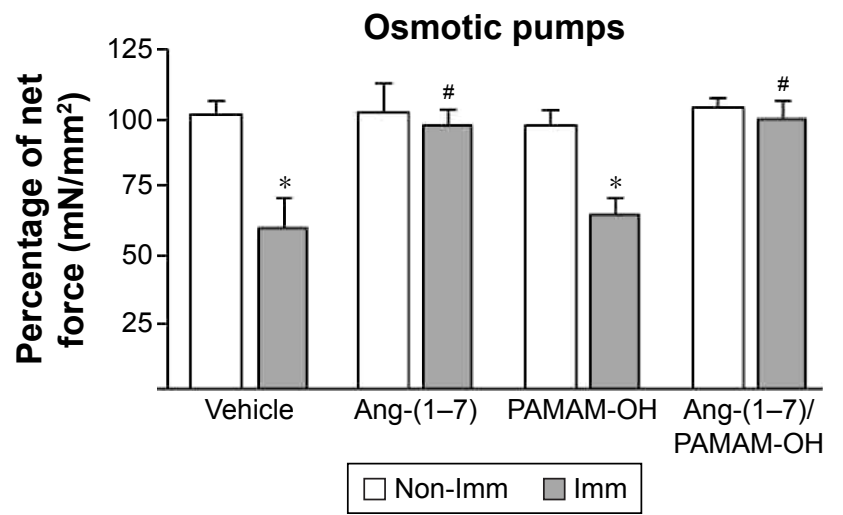

Figure S2 Systemic administration of Ang-(I-7) alone and Ang-(I-7)/PAMAM-OH complex by osmotic pumps prevented the decrease in isometric force of immobilized muscle. Notes: Osmotic pumps were implanted in mice with vehicle, Ang-(I-7), PAMAM-OH or Ang-(I-7)/PAMAM-OH complex and unilaterally immobilized for I4 d. GAST muscles non-immobilized (Non-Imm) or contralateral immobilized $(\mathrm{Imm})$ were excised, and the maximal isometric strength $\left(\mathrm{mN} / \mathrm{mm}^{2}\right)$ was evaluated for each condition. Values represent the mean $\pm S D$ of three independent experiments. In each experiment, five to seven mice were used for each experimental condition. $(* P<0.05$ versus vehicle-treated/non-immobilized GAST; ${ }^{*} P<0.05$ versus vehicle-treated/immobilized GAST.)

Abbreviations: Ang-(I-7), angiotensin (I-7); PAMAM-OH, hydroxyl-terminated poly(amidoamine); GAST, gastrocnemius; SD, standard deviation. 


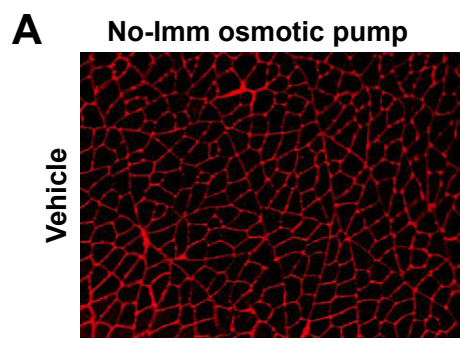

\section{Imm osmotic pump}
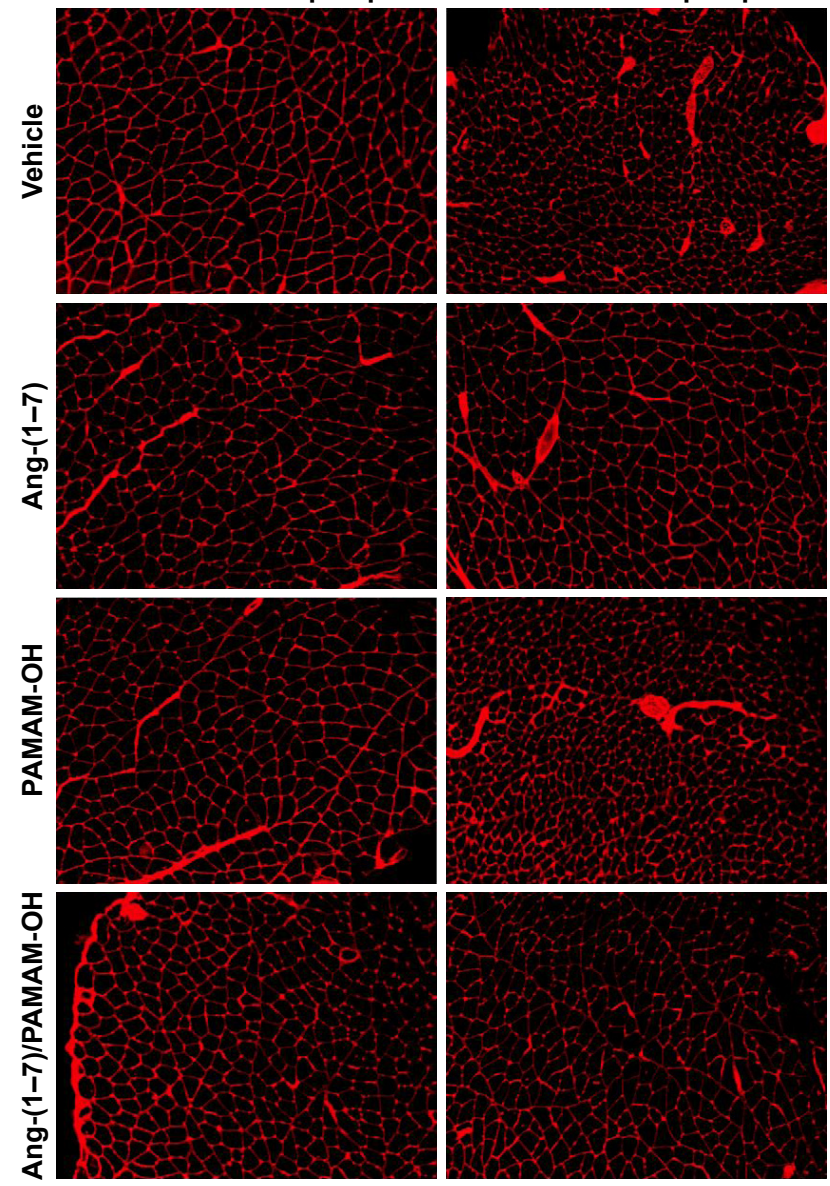

B
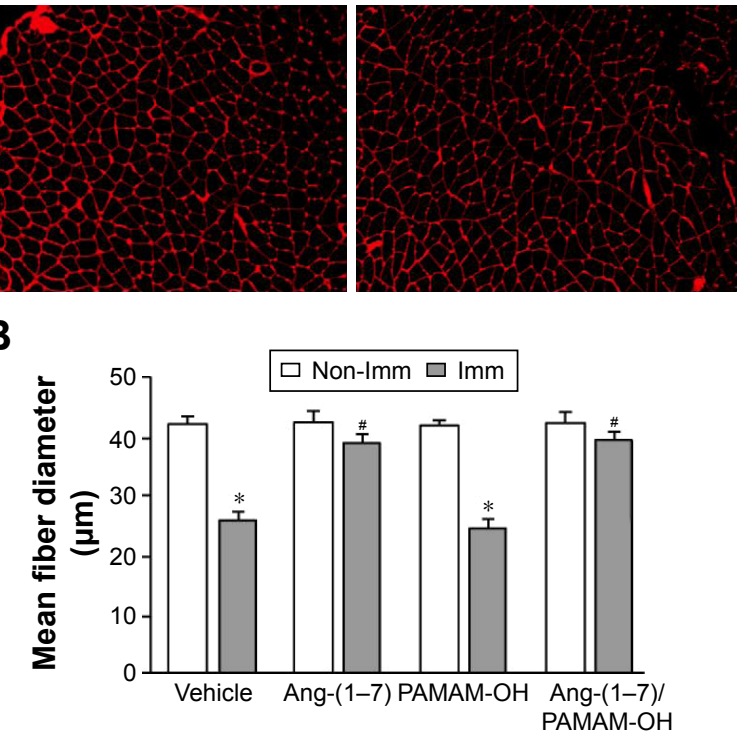

Figure S3 Ang-(I-7) alone and Ang-( I-7)/PAMAM-OH complex systemically administered by osmotic minipumps, recovered the fiber diameter in immobilized muscles. Notes: The fiber diameter of GAST muscles non-immobilized (Non-Imm) or contralateral hind limb immobilized for I4 d (Imm) from mice previously implanted with osmotic pumps containing vehicle, Ang-(I-7), PAMAM-OH, or Ang-(I-7)/PAMAM-OH complex that were excised and rapidly froze. (A) Muscle cross-sections were stained with WGA to delimit muscle fiber sarcolemma. (B) Minimal Feret diameters of Non-imm and Imm (with osmotic pump) were determined in the GAST cross-sections from (A). The values are expressed as the mean fiber diameter. The graph represents the quantification of three independent experiments. Five to seven mice were used for each experimental

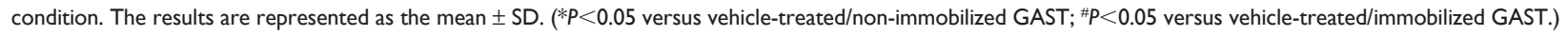

Abbreviations: Ang-(I-7), angiotensin (I-7); PAMAM-OH, hydroxyl-terminated poly(amidoamine); GAST, gastrocnemius; WGA, wheat germ agglutinin; SD, standard deviation. 
A

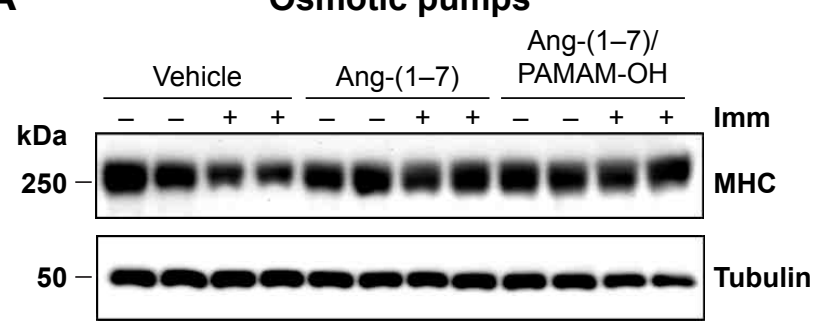

C

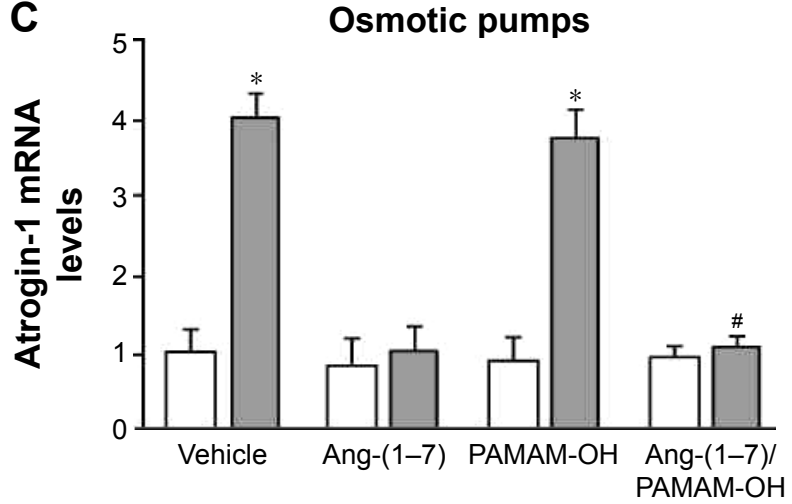

B

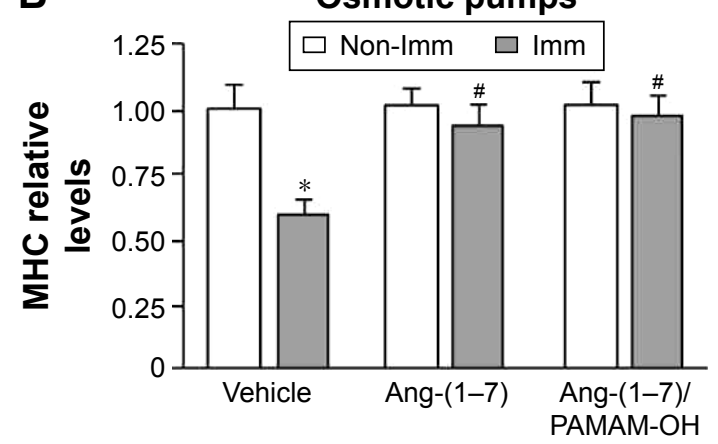

D Osmotic pumps

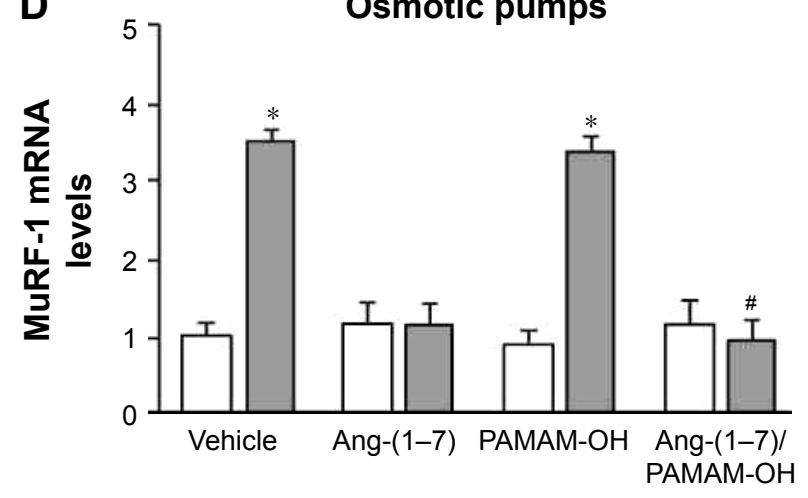

$\square$ Non-Imm $\square \mathrm{Imm}$

Figure S4 Systemic administration of Ang-(I-7) alone and Ang-(I-7)/PAMAM-OH complex avoided the diminution of MHC levels and the upregulation of atrogin-I and MuRF-I expression in atrophied muscle by disuse.

Notes: Mice implanted with osmotic pumps containing vehicle, Ang-(I-7), or Ang-(I-7)/PAMAM-OH complex were unilaterally immobilized for I4 d. GAST muscles of hind limb non-immobilized (Non-Imm) and immobilized ( $\mathrm{Imm}$ ) were extracted. (A) Proteins extracts of homogenized muscles were made to evaluate MHC protein levels through Western blot analysis. Tubulin levels were used as the loading control, and molecular weight markers are shown in kilodaltons. (B) Quantitative analysis of the bands by densitometry was performed, and the levels of MHC were normalized to tubulin. The results are expressed relative to vehicle with pump/non-immobilized GAST and correspond to the mean $\pm S D$ from triplicate independent experiments. In each experiment, five to seven mice were used for each experimental condition. $(* P<0.05$ versus vehicle-treated/non-immobilized GAST; ${ }^{P}<0.05$ versus vehicle-treated/immobilized GAST.) Total RNA were extracted and atrogin-I (C) and MuRF-I (D) levels were detected through RT-qPCR using tubulin as the reference gene. Expression is shown as the fold of induction relative to vehicle-treated/non-immobilized GAST. The values correspond to the mean \pm SD of triplicate independent experiments. In each experiment, five to seven mice were used for each experimental condition $(* P<0.05$ versus vehicle-treated/non-immobilized GAST; ${ }^{*} P<0.05$ versus vehicle-treated/immobilized GAST.)

Abbreviations: Ang-(I-7), angiotensin (I-7); PAMAM-OH, hydroxyl-terminated poly(amidoamine); MHC, myosin heavy chain; GAST, gastrocnemius; SD, standard deviation; RT-qPCR, reverse transcription quantitative real-time polymerase chain reaction.

\section{Publish your work in this journal}

The International Journal of Nanomedicine is an international, peerreviewed journal focusing on the application of nanotechnology in diagnostics, therapeutics, and drug delivery systems throughout the biomedical field. This journal is indexed on PubMed Central, MedLine, CAS, SciSearch ${ }^{\circledR}$, Current Contents ${ }^{\circledR} /$ Clinical Medicine,
Journal Citation Reports/Science Edition, EMBase, Scopus and the Elsevier Bibliographic databases. The manuscript management system is completely online and includes a very quick and fair peer-review system, which is all easy to use. Visit http://www.dovepress.com/ testimonials.php to read real quotes from published authors. 